\title{
Geodesics of an affine connection and electromagnetism with radiation reaction
}

\author{
R. R. Burman
}

\begin{abstract}
This paper deals with the motion of a point test charge in an external electromagnetic field with the effect of electromagnetic radiation reaction included. The equation of motion applicable in a general Riemannian space-time is written as the geodesic equation of an affine connection. The connection is the sum of the Christoffel connection and a tensor which depends on, amons other things, the external electromagnetic field, the charge and mass of the particle and the Ricci tensor. The affinity is not unique; a choice is made so that the covariant derivative of the metric tensor with respect to the connection vanishes. The special cases of conformally flat spaces and the space of general relativity are discussed.
\end{abstract}

\section{Introduction}

The generally covariant equation of motion of a test charge in an external electromagnetic field, with radiation reaction neglected, has been written as the equations of geodesics in a Finsler space and in a five-dimensional Riemannian space [18]. It has also been expressed, within the usual four-dimensional Riemannian space, in the form of the geodesic equation of an affine connection [7]; the affinity is the sum of the Christoffel connection and a third rank tensor which depends on the electromagnetic field and on the 4-velocity and charge-to-mass ratio of the particle. The affinity is not unique, there being a class of

Received 5 November 1970. 
affinities having the same geodesics, but can be chosen so that the covariant derivative of the metric tensor, taken with respect to the affinity, vanishes [7]; this property is preserved if any further skew part with zero torsion vector is added to the affinity.

The equations of motion of general relativity, for a test particle under forces arising from fields with arbitrary energy-momentum tensors, can also be expressed as the geodesic equations of an affine connection satisfying the condition mentioned above [2].

In the present paper, the work of Droz-Vincent [7] is extended to include the effect of the force of electromagnetic radiation reaction; gravitational radiation reaction is still neglected. As a preliminary step, the generally covariant form of the equation of motion used in special relativity, namely the Lorentz-Dirac equation, is expressed as a geodesic equation. Then the results are extended to the equation derived by DeWitt and Brehme [4] and Hobbs [11] for motion in a general four-dimensional Riemannian space-time.

\section{The generally covariant Lorentz-Dirac equation}

Consider a charged point particle, of charge $e$ and mass $m$, acted on by an external electromagnetic field $\left(\phi_{\mu \nu}\right)$, in the Minkowski space of special relativity. Let $\left(x^{\mu}\right)$ denote the space-time position $(r$, ct) of the particle, $c$ being the speed of light in empty space. The equation of motion with the effect of radiation reaction included is the Lorentz-Dirac equation $[6 ; 1, \mathrm{p} .196 ; 14]$ :

$$
m a^{\mu}=\frac{e}{c} \phi^{\alpha \mu} u_{\alpha}+\frac{2 e^{2}}{3 c^{3}}\left(\dot{a}^{\mu}+\frac{1}{c^{2}} a_{\lambda} a^{\lambda} u^{\mu}\right) \text {. }
$$

In (I), $\left(u^{\mu}\right)$ and $\left(a^{\mu}\right)$ are the 4-velocity and 4-acceleration of the charge: $u^{\mu}=\dot{x}^{\mu}$ and $a^{\mu}=\ddot{x}^{\mu}$, where a dot denotes differentiation with respect to proper time. Also, $u_{v} u^{v}=c^{2}$.

Equation (1) can be written in generally covariant form by replacing the partial derivatives by covariant ones. If $d s$ denotes the Riemannian space-time interval, then $u^{\mu}=\dot{x}^{\mu}=c d x^{\mu} / d s$, and using the general 
expression for the covariant derivative of a vector, it follows that

$(2 a, b)$

$$
a^{\mu}=c u_{; \nu}^{\mu} \frac{d x^{\nu}}{d s}=c^{2}\left(\frac{d^{2} x^{\mu}}{d s^{2}}+\left\{\begin{array}{c}
\mu \\
\alpha \beta
\end{array}\right\} \frac{d x^{\alpha}}{d s} \frac{d x^{\beta}}{d s}\right)
$$

and

$$
\dot{a}^{\mu}=c^{3}\left(\frac{d^{3} x^{\mu}}{d s^{3}}+\left\{\begin{array}{c}
\mu \\
\alpha^{\beta} \beta
\end{array}\right\} \frac{d^{2} x^{\alpha}}{d s^{2}} \frac{d x^{\beta}}{d s}\right) .
$$

Here, a dot denotes absolute covariant differentiation with respect to proper time, while a semi-colon denotes covariant differentiation with respect to the Christoffel connection, which is represented by the braces.

The skew tensor $\left(s^{\alpha \beta}\right)$ is defined by [14]

$$
s^{\alpha \beta}=\frac{2 e}{3 c^{4}}\left(u^{\alpha} a^{\beta}-a^{\alpha} u^{\beta}\right) \text {. }
$$

In Riemannian space $d s^{2}=g_{\mu \nu} d x^{\mu} d x^{\nu},\left(g_{\mu \nu}\right)$ being the metric tensor, which will be used to lower and raise indices. So $u_{v} u^{v}=c^{2}$, which implies that $a^{v} u_{v}=0$ and hence $\dot{a}^{v u_{v}}=-a_{v} a^{v}$. Using these together with (4), it follows that

$$
\dot{s}^{\beta \mu} u_{\beta}=\frac{2 e}{3 c^{2}}\left(\dot{a}^{\mu}+\frac{1}{c^{2}} \dot{a}_{\lambda} a^{\lambda} u^{\mu}\right)
$$

- the 4-force of radiation reaction is equal to $\left(e \dot{s}^{\beta \mu} u_{\beta} / c\right)$. So, using $(2 b)$, the generally covariant form of the Lorentz-Dirac equation can be written

$$
\frac{d^{2} x^{\mu}}{d s^{2}}+\left\{\begin{array}{c}
\mu \\
\alpha \beta
\end{array}\right\} \frac{d x^{\alpha}}{d s} \frac{d x^{\beta}}{d s}=\frac{e}{m c^{3}}\left(\phi^{\alpha \mu}+\dot{s}^{\alpha \mu}\right) u_{\alpha} .
$$

The quantities defined by

$$
\Delta_{\alpha \beta}^{\mu}=\left\{\begin{array}{c}
\mu \\
\alpha \beta
\end{array}\right\}+A_{\alpha \beta}^{\mu},
$$

where 


$$
A_{\alpha \beta}^{\mu}=\frac{-e}{m c^{3}}\left(\phi_{\alpha}^{\mu}+\dot{s}_{\alpha}^{\mu}\right) u_{\beta},
$$

form an affine connection $\Delta$, since the sum of an affinity and a tensor of the appropriate type is another affinity. Since $u_{\beta} u^{\beta}=c^{2}$, it is seen that (6) can be written

$$
\frac{d^{2} x^{\mu}}{d s^{2}}+\Delta_{\alpha \beta}^{\mu} \frac{d x^{\alpha}}{d s} \frac{d x^{\beta}}{d s}=0
$$

- the geodesic equation of $\Delta$. When radiation reaction is neglected, the connection $\Delta$ reduces to that introduced previously [7].

From (2a), (6) can also be expressed as

$$
u^{\nu} u_{; \nu}^{\mu}=\frac{e}{m c}\left(\phi^{\alpha \mu}+\dot{s}^{\alpha \mu}\right) u_{\alpha}
$$

Letting a dot denote covariant differentiation with respect to $\Delta$, (9) can be expressed as

$$
u^{\nu} u^{\mu} \cdot v=0
$$

- an alternative form of the geodesic equation.

From the general expression for a covariant derivative, using (7),

$$
g_{\mu \nu, \rho}=g_{\mu \nu ; \rho}-A_{\mu \nu \rho}-A_{\nu \mu \rho} \text {. }
$$

Since $g_{\mu \nu ; \rho}=0$ and since $\left(\phi_{\alpha \beta}\right)$ and $\left(s_{\alpha \beta}\right)$ are skew-symmetric, it follows that $g_{\mu \nu . \rho}=0$.

Square brackets around a pair of indices will denote a skew part. Because of the skew-symmetry of $\left(\phi_{\mu \nu}\right)$ and $\left(\dot{s}_{\mu \nu}\right), \phi_{\sigma}^{\sigma}$ and $\dot{s}_{\sigma}^{\sigma}$ vanish; hence the torsion vector of $\Delta$ has components given by

$$
\Delta_{\alpha} \equiv \Delta_{[\alpha \quad \sigma]}^{\sigma}=\frac{-e}{2 m c^{3}}\left(\phi_{\alpha}^{\sigma}+\dot{s}_{\alpha}^{\sigma}\right) u_{\sigma}
$$

and so is proportional to the total 4-force (external plus radiation reaction) acting on the particle.

The above connection is not unique, but has been chosen to give 
$g_{\mu v, \rho}=0$. If $\left(V_{\alpha}\right)$ is a vector and $\left(B_{\alpha, \beta}^{\mu}\right)$ a skew-symmetric third rank tensor, the quantities

$$
\Delta_{\alpha \beta}^{\prime \mu}=\Delta_{\alpha \beta}^{\mu}+\delta_{\alpha}^{\mu} V_{\beta}+\delta_{\beta}^{\mu} V_{\alpha}+B_{\alpha \beta}^{\mu}
$$

form a connection with the same geodesics as $\Delta$ [16]. If $V_{\alpha}=0$ and the torsion vector of $B$ vanishes, $g_{\mu \nu . \rho}=0$ holds with respect to $\Delta^{\prime}$ if it does with respect to $\Delta$ [7]. The geodesics of an affinity are unaffected by its skew part. The affinity $\Delta$ defined by (7) and (8) is non-symmetric, as it must be for $g_{\mu v . \rho}=0$ to hold: a tensor $B$ chosen to cancel the skew part would have a non-zero torsion vector.

\section{Motion in a general Riemannian space-time}

Dewitt and Brehme [4] investigated the motion of a charged particle in an external electromagnetic field in a Riemannian space-time of arbitrary (hyperbolic) metric; they found the equation of motion to be not just the generally covariant form of (I) but to have an additional term

$$
\frac{e^{2}}{c} u_{\alpha} \int_{-\infty}^{\tau} f_{B}^{\mu \alpha}, u^{\beta^{\prime}}\left(\tau^{\prime}\right) d \tau^{\prime}
$$

on the right. Here $\tau$ is the proper time and $\left(f^{\mu \nu} \beta^{\prime}\right)$ is a bi-tensor which is skew in its first two indices. The additional force acting on the charge is non-local in time, and arises from fields originating from the charge and propagated back by the scattering effects of the space-time curvature: Huygens' principle fails in curved space-time $[4,5]$.

The problem was further investigated by Hobbs [11], who found that yet another term should be included; this term also arises from the scattering of the electromagnetic fields due to the space-time curvature. The equation of motion takes the form of (1) with both (13) and

$$
\frac{-e^{2}}{3 c}\left(R^{\alpha \mu}-\frac{1}{e^{2}} R^{\alpha \tau} u_{\tau} u^{\mu}\right) u_{\alpha}
$$

added to the right side; $\left(R^{\alpha \beta}\right)$ is the Ricci tensor (the contracted Riemann-Christoffel curvature tensor), defined in terms of the Christoffel 
connection.

The equation of motion can be written in the form of (6) with the bracketed quantity on the right side replaced by

$$
\phi^{\alpha \mu}+\dot{s}^{\alpha \mu}+t^{\alpha \mu}+r^{\alpha \mu}
$$

where

$$
t^{\alpha \mu}=e \int_{-\infty}^{\tau} f_{B^{\prime}}^{\mu \alpha} u^{\beta^{\prime}}\left(\tau^{\prime}\right) d \tau^{\prime}
$$

and

$$
r^{\alpha \mu}=\frac{-e}{3}\left(R^{\alpha \mu}-\frac{1}{c^{2}} R^{\alpha \tau} u_{\tau} u^{\mu}\right)
$$

Since $\left(f^{\mu \nu}{ }^{\prime}\right)$ is skew in its first two indices, $\left(t^{\mu \nu}\right)$ is skew.

The quantities defined by (7), where now

$$
A_{\alpha \beta}^{\mu}=\frac{-e}{m c^{3}}\left(\phi_{\alpha}^{\mu}+\dot{s}_{\alpha}^{\mu}+t_{\alpha}^{\mu}+r_{\alpha}^{\mu}\right) u_{B}
$$

form an affine connection $\Delta$, and the equation of motion can be written as the geodesic equation of $\Delta$, namely (9).

The torsion vector of $\Delta$ now has components given by

$$
\Delta_{\alpha} \equiv \Delta_{[\alpha \sigma]}^{\sigma}=\frac{-e}{2 m c^{3}}\left[\left(\phi_{\alpha}^{\sigma}+\dot{s}_{\alpha}^{\sigma}+t_{\alpha}^{\sigma}+r_{\alpha}^{\sigma}\right) u_{\sigma}-r_{\sigma}^{\sigma} u_{\alpha}\right] \text {. }
$$

Because $\left(r_{\mu \nu}\right)$ is not skew-symmetric, $\left(\Delta_{\alpha}\right)$ is not proportional to the 4-force acting on the particle. From (17),

$$
r_{\sigma}^{\sigma}=\frac{-e}{3}\left(R-\frac{1}{c^{2}} R_{\sigma \tau} u^{\sigma} u^{\tau}\right)
$$

in which $R$ is the curvature scalar $R_{\sigma}{ }^{\sigma}$.

The fact that $\left(r_{\mu \nu}\right)$ is not skew-symmetric also means that $g_{\mu \nu . \rho}$ does not vanish. With $(18),(10)$ becomes, since $g_{\mu \nu ; p}=0$,

$$
g_{\mu \nu . \rho}=\frac{e}{m c^{3}}\left(r_{\mu \nu}+r_{\nu \mu}\right) u_{\rho} .
$$


So, using (17),

$$
g_{\mu \nu, \rho}=\frac{-2 e^{2}}{3 m c^{3}}\left(R_{\mu \nu}-\frac{1}{c^{2}} R\left(\mu u_{\nu}\right)_{\tau} u_{\rho}\right.
$$

where round brackets enclosing a pair of indices denote the symmetric part. An alternative connection, with respect to which the covariant derivative of $\left(g_{\mu \nu}\right)$ vanishes, can be found as follows.

The equation of motion can be written

(23) $\frac{d^{2} x^{\mu}}{d s^{2}}+\left\{\begin{array}{c}\mu \\ \alpha \beta\end{array}\right\} \frac{d x^{\alpha}}{d s} \frac{d x^{\beta}}{d s}=$

$$
\frac{e}{m c^{3}}\left\{\phi^{\alpha \mu}+\dot{s}^{\alpha \mu}+t^{\alpha \mu}\right) u_{\alpha}-\frac{e^{2}}{3 m c^{3}}\left(R^{\alpha \mu} u_{\alpha}-\frac{1}{c^{2}} R^{\alpha \tau} u_{\alpha} u_{\tau} u^{\mu}\right)
$$

with $\left(s^{\alpha \beta}\right)$ and $\left(t^{\alpha \beta}\right)$ defined by (4) and (16). Consider the affine connection $\Gamma$ defined by

$$
\Gamma_{\alpha \beta}^{\mu}=\left\{\begin{array}{c}
\mu \\
\alpha \beta
\end{array}\right\}+B_{\alpha \beta}^{\mu}
$$

where

$$
B_{\alpha \beta}^{\mu}=\frac{-e}{m c^{3}}\left(\phi_{\alpha}^{\mu}+\dot{s}_{\alpha}^{\mu}+t_{\alpha}{ }^{\mu}\right) u_{B}+B_{\alpha}{ }^{\mu} u_{\beta}
$$

in which $\left(B_{\alpha \beta}\right)$ is a skew-symmetric second rank tensor. Since $\left(B_{\alpha \mu \beta}\right)$ is skew-symmetric in its first two indices, it is seen from (10) that $g_{\mu \nu . \rho}=0$ where a dot now denotes covariant differentiation with respect to $\Gamma$. Equation (23) can be expressed as the geodesic equation of $\Gamma$ provided

$$
B_{\alpha}{ }^{\mu} u^{\alpha}=\frac{e^{2}}{3 m c^{3}}\left(R^{\alpha \mu} u_{\alpha}-\frac{1}{c^{2}} R^{\alpha \tau} u_{\alpha} u_{\tau} u^{\mu}\right) \text {. }
$$

This condition is satisfied by the skew tensor $\left(B_{\mu \nu}\right)$, where

$$
B_{\mu v}=\frac{2 e^{2}}{3 m c^{5}} u_{[\mu}^{R}{ }_{v] \tau} u^{\tau}
$$

With (27), the components of $\Gamma$ are given by 


$$
\Gamma_{\alpha \beta}^{\mu}=\left\{\begin{array}{c}
\mu \\
\alpha \beta
\end{array}\right\}-\frac{e}{m c^{3}}\left(\phi_{\alpha}^{\mu}+\dot{s}_{\alpha}^{\mu}+t_{\alpha}^{\mu}\right) u_{\beta}+\frac{e^{2}}{3 m c^{5}}\left(u_{\alpha} R^{\mu}{ }_{\tau}-R_{\alpha \tau} u^{\mu}\right) u^{\tau} u_{\beta} .
$$

The torsion vector of $\Gamma$. has components given by

$$
\Gamma_{\alpha} \equiv \Gamma_{[\alpha \sigma]}^{\sigma}=\frac{-e}{2 m c^{3}}\left(\phi_{\alpha}^{\sigma}+\dot{s}_{\alpha}^{\sigma}+t_{\alpha}^{\sigma}\right) \dot{u}_{\sigma}-\frac{e^{2}}{6 m c^{3}}\left(R_{\alpha \tau}-\frac{1}{c^{2}} u_{\alpha} R_{\sigma \tau} u^{\sigma}\right) u^{\tau}
$$

and so is proportional to the total 4-force acting on the particle.

\section{Motion in a conformally flat space-time}

A conformally flat space is one in which $d s^{2}$ has the form $\exp (2 \Omega)$ $\exp (2 \Omega) n_{\nu \nu} d x^{\mu} d x{ }^{\nu}$ where $\left(n_{\mu \nu}\right)$ is the rlat space metric tensor and $\Omega$ is a function of the $x^{\mu}$. In such space-times, the force term found by DeWitt and Brehme vanishes [12]. Also [19, Chapter 8]

$$
R_{\mu \nu}=2\left(\Omega_{, \mu \nu}-\Omega_{, \mu, \nu}\right)+\eta_{\mu \nu} \eta^{\alpha \beta}\left(\Omega_{, \alpha \beta}+2 \Omega_{, \alpha} \Omega_{, \beta}\right)
$$

and

$$
R=6 e^{-2 \Omega_{n}^{\alpha \beta}}\left(\Omega, \alpha \beta+\Omega, \Omega_{, \beta}\right)
$$

where a comma denotes partial differentiation.

The force term found by Hobbs, namely (14), can be written

$$
\frac{-e^{2}}{3 c} R_{\alpha \tau}\left(g^{\mu \tau}-\frac{1}{c^{2}} u^{\mu} u^{\tau}\right) u^{a} \text {. }
$$

In a conformally flat space, using (30) and the fact that

$$
\eta_{\alpha \tau}\left(g^{\mu \tau}-\frac{1}{c^{2}} u^{\mu} u^{\tau}\right) u^{\alpha}
$$

vanishes, (32) becomes

$$
\frac{-2 e^{2}}{3 c}\left(\Omega, \alpha \tau-\Omega, a^{\Omega}, \tau\right)\left(g^{\mu \tau}-\frac{1}{c^{2}} u^{\mu} u^{\tau}\right) u^{\alpha}
$$

where $g^{\mu \tau}=\exp (-2 \Omega) \eta^{\mu \tau}$.

The equation of motion can be expressed as the geodesic equation of the affine connection defined by $(7)$ and (18), with $\left\{r_{\alpha}^{\mu}\right)$ replaced by 
$\left(v_{\alpha}^{\mu}\right)$ where

$$
v_{\alpha}^{\mu}=\frac{-2 e}{3}\left(\Omega, \alpha \tau-\Omega, \alpha^{\Omega}, \tau\right)\left(g^{\mu \tau}-\frac{1}{e^{2}} u^{\mu} u^{\tau}\right)
$$

Alternatively, the connection can be defined by (24) and (25) where now, using (30) in (27),

$$
\left.\left.B_{\mu \nu}=\frac{4 e^{2}}{3 m c^{5}} u_{[\mu}(\Omega, \nu] \tau-\Omega, \nu\right]^{\Omega}, \tau\right) u^{\tau} .
$$

Then

$$
\text { (37) } \begin{aligned}
\Gamma_{\alpha \beta}^{\mu}=\left\{\begin{array}{c}
\mu \\
\alpha \beta
\end{array}\right\} & -\frac{e}{m c^{3}}\left(\phi_{\alpha}^{\mu}+\dot{s}_{\alpha}^{\mu}+t_{\alpha}{ }^{\mu}\right) u_{\beta} \\
& +\frac{2 e^{2}}{3 m c^{5}}\left[u_{\alpha}\left(\Omega^{\mu}, \tau-\Omega^{\mu}{ }^{\mu}, \tau\right)-u^{\mu}\left(\Omega, \alpha \tau-{ }^{\mu}, \alpha^{\Omega}, \tau\right)\right] u^{\tau} u_{B}
\end{aligned}
$$

and the torsion vector has the components

$$
\begin{aligned}
\text { (38) } \Gamma_{\alpha}=\frac{-e}{2 m c^{3}}\left(\phi_{\alpha}{ }^{\sigma}+\dot{s}_{\alpha}{ }^{\sigma}+t_{\alpha}{ }^{\sigma}\right) u_{\sigma} \\
\quad-\frac{e^{2}}{3 m c^{3}}\left[\Omega, \alpha \tau-\Omega, \alpha^{\Omega}, \tau-\frac{1}{c^{2}} u_{\alpha} u^{\sigma}\left(\Omega, \sigma \tau-\Omega, \sigma, \sigma^{\Omega}\right)\right] u^{\top} .
\end{aligned}
$$

For a certain class of conformally flat space-times, the force term (34) vanishes [12]. The condition for this is $\Omega, \alpha \beta=\Omega, \Omega_{, \beta}$, the solution of which is [12]

$$
e^{\Omega}=\frac{1}{a_{\alpha} x^{\alpha}+b}
$$

where $\left(a_{\alpha}\right)$ is an arbitrary constant 4 -vector and $b$ is an arbitrary constant. In such a space-time, the motion is described by the generally covariant form of the Lorentz-Dirac equation. If the space-time is required to be spatially isotropic, (39) becones [12] $\exp (\Omega)=1 / H t, H$ being constant: this corresponds to the de Sitter metric, as used in the de sitter universe (which is empty, stationary and has a cosmological constant equal to $3 H^{2} / c^{2}$ ) and in the steady-state cosmology. 


\section{Application of Einstein's field equations}

As seen in the previous sections, the force term discovered by Hobbs [11] introduces into the affine connection a part which involves the Ricci tensor $\left(R_{\mu \nu}\right)$. In this section, Einstein's field equations of general relativity, which have not so far been used, will be introduced and used to express the geometrical quantities $\left(R_{\mu \nu}\right)$ and $R$ in terms of physical quantities.

The field equations are

$$
R_{\mu}^{\nu}+\left(\lambda-\frac{1}{2} R\right) \delta_{\mu}{ }^{\nu}=-\kappa\left(M_{\mu}{ }^{\nu}+T_{\mu}{ }^{\nu}\right)
$$

where $\lambda$ is the cosmological constant and $\kappa$ is a constant, while $\left(M_{\mu v}\right)$ and $\left(T_{\mu \nu}\right)$ are the energy-momentum tensors of matter and the non-gravitational fields, respectively. Contracting (40) gives

$$
R-4 \lambda=\kappa(M+T)
$$

where $M \equiv M_{\sigma}^{\sigma}$ and $T \equiv T_{\sigma}{ }^{\sigma}$. Use of (41) to eliminate $R$ from (40) gives

$$
R_{\mu}^{\nu}-\lambda \delta_{\mu}^{\nu}=-\kappa\left(M_{\mu}^{\nu}-\frac{1}{2} M \delta_{\mu}^{\nu}\right)-\kappa\left(T_{\mu}^{\nu}-\frac{1}{2} T \delta_{\mu}^{\nu}\right) .
$$

For incoherent matter (dust), of proper density $\rho, M_{\mu}{ }^{\nu}=\rho u_{\mu} u^{\nu}$ and $M=\rho c^{2}$; these formulas can be applied to a single test particle by writing $\rho(x)=m \delta(x-\bar{x}) / \sqrt{-g}$ where $\delta$ is a four-dimensional delta function, $x$ and $\bar{x}$ represent points in space-time, the latter being on the particle's world line, and $g \equiv \operatorname{det}\left(g_{\mu \nu}\right)$ [17]. If $\left(T_{\mu}^{\nu}\right)$ represents the electromagnetic field, then $T=0$ [8, p. 183]. So, for a point test particle in the presence of an electromagnetic field, (41) and (42) become

$$
R-4 \lambda=\frac{k m c^{2}}{\sqrt{-g}} \delta(x-\bar{x})
$$

and 


$$
R_{\mu}^{\nu}-\lambda \delta_{\mu}^{\nu}=-\kappa T_{\mu}^{\nu}-\frac{k m}{\sqrt{-g}} \delta(x-\bar{x})\left(u_{\mu} u^{\nu}-\frac{c^{2}}{2} \delta_{\mu}^{\nu}\right) .
$$

Equation (44) gives

$$
R_{\alpha \tau} u^{\tau}=-\kappa T_{\alpha \tau} u^{\tau}+\left(\lambda-\frac{k m c^{2}}{2 \sqrt{-g}} \delta(x-\bar{x})\right) u_{\alpha}
$$

and hence

$$
\frac{1}{c^{2}} R_{\sigma \tau} u^{\sigma} u^{\tau}=\frac{-k}{c^{2}} T_{\sigma \tau} u^{\sigma} u^{\tau}+\lambda-\frac{\mathrm{km} c^{2}}{2 \sqrt{-g}} \delta(x-\bar{x}) .
$$

Equations (45) and (46) show that

$$
\frac{-e^{2}}{3 c}\left(R_{\alpha \tau}-\frac{1}{c^{2}} u_{\alpha} R \sigma u^{\sigma}\right) u^{\tau}=\frac{e^{2} \kappa}{3 c}\left(T_{\alpha \tau}-\frac{1}{c^{2}} u_{\alpha} T_{\sigma \tau} u^{\sigma}\right) u^{\tau} \text {. }
$$

So, assuming Einstein's field equations to be valid, the components of the force term found by Hobbs [11] can be expressed as the right side of (47).

Equations (17), (20) and (27) can be written, using (43) to (46),

$$
\begin{gathered}
r_{\alpha}^{\mu}=\frac{-e}{3}\left[\left(\lambda+\frac{\kappa m c^{2}}{2 \sqrt{-g}} \delta(x-\bar{x})\right)\left(\delta_{\alpha}^{\mu}-\frac{1}{c^{2}} u_{\alpha} u^{\mu}\right)-\kappa\left(T_{\alpha}^{\mu}-\frac{1}{c^{2}} T_{\alpha \tau} u^{\top} u^{\mu}\right)\right], \\
r_{\sigma}^{\sigma}=-e\left[\lambda+\frac{\kappa m c^{2}}{2 \sqrt{-g}} \delta(x-\bar{x})+\frac{\kappa}{3 c^{2}} u^{\sigma} u^{\tau} T_{\alpha \tau}\right]
\end{gathered}
$$

and

$$
B_{\mu \nu}=\frac{-2 e^{2} \kappa}{3 m c^{5}} u_{\left[\mu^{T}\right] \tau^{T}} u^{\tau}
$$

It is noted that $\left(B_{\alpha \beta}\right)$, unlike $\left(r_{\alpha \beta}\right)$, does not contain the cosmological constant or any delta function terms.

The electromagnetic energy-momentum tensor has components

$$
T_{\mu}^{\nu}=\frac{1}{4 \pi}\left(\phi_{\mu \beta} \phi^{\beta \nu}+\frac{1}{4} \delta_{\mu}{ }^{\nu} \phi_{\sigma \tau} \phi^{\sigma \tau}\right) \text {, }
$$

so the right side of (47) can be written

$$
\frac{e^{2} \kappa}{12 \pi c}\left(\phi_{\alpha \beta}-\frac{1}{c^{2}} u_{\alpha} \phi_{\alpha \beta} u^{\sigma}\right) \phi^{\beta \tau} u_{\tau},
$$

which expresses the components of Hobb's 4-force in terms of the 
electromagnetic field tensor. The external 4 -force $\left(k_{v}\right)$ acting on the test charge has components

$$
k_{v}=\frac{e}{c} \phi_{\alpha v^{u}}{ }^{\alpha}
$$

so (52) can be written

$$
\frac{-e k}{12 \pi}\left(\phi_{\alpha \beta}-\frac{1}{c^{2}} u_{\alpha} \phi_{\sigma \beta} u^{\sigma}\right) k^{\beta} .
$$

Equation (50) can be expressed as

$$
B_{\mu \nu}=\frac{e^{2} K}{6 \pi m c^{5}} u_{[\mu} \phi_{\nu] \alpha^{\phi}}{ }^{\lambda \alpha} u_{\lambda}
$$

or as

$$
B_{\mu \nu}=\frac{e k}{6 \pi m c^{4}} u_{[\mu} \phi_{\nu] \alpha} k^{\alpha}
$$

Thus, when the field equations of general relativity are used, the equation of motion can be expressed as the geodesic equation of the affine connection $\Gamma$ defined by

(57) $\Gamma_{\alpha \beta}^{\mu}=$

$$
\left\{\begin{array}{c}
{ }_{\alpha \beta}^{\mu} \\
\alpha^{\prime}
\end{array}-\frac{e}{m c^{3}}\left(\phi_{\alpha}^{\mu}+\dot{s}_{\alpha}^{\mu}+t_{\alpha}^{\mu}\right) u_{\beta}+\frac{e^{2} \kappa}{12 \pi m c^{5}}\left(u_{\alpha} \phi_{\tau}^{\mu}-\phi_{\alpha \tau} u^{\mu}\right) \phi^{\lambda \tau} u_{\lambda} u_{\beta}\right. \text {. }
$$

Because of the skew-symmetry of $\left(\phi_{\mu \nu}\right),\left(\dot{s}_{\mu \nu}\right),\left(t_{\mu \nu}\right)$ and $\left(B_{\mu \nu}\right)$, the covariant derivative of $\left(g_{\mu \nu}\right)$ with respect to $\Gamma$ vanishes. The torsion vector has components

(58) $\Gamma_{\alpha}=\frac{-e}{2 m c^{3}}\left(\phi_{\alpha}^{\sigma}+\dot{s}_{\alpha}^{\sigma}+t_{\alpha}^{\sigma}\right) u_{\sigma}-\frac{e^{2} \kappa}{24 \pi m c^{3}}\left(\phi_{\alpha \tau}-\frac{1}{e^{2}} u_{\alpha} \phi_{\sigma \tau} u^{\sigma}\right) \phi^{\lambda \tau} u_{\lambda}$ and so is proportional to the total 4-force on the charge.

\section{Rohrlich's electrodynamics}

To overcome certain formal difficulties (divergences) which arise in classical electrodynamics, Rohrlich [13, 14] proposed a new approach. He developed a theory of the interaction of charged point particles which is 
Lorentz covariant but neglects quantum effects; radiation reaction is included, and the theory leads to the Lorentz-Dirac equation of motion. The theory was generalized to apply in a Riemannian space-time $[14,15]$, and the equation of motion for a test charge was then found to be (in slightly different notation)

$$
\frac{d^{2} x^{\mu}}{d s^{2}}+\left\{\begin{array}{c}
\mu \\
\alpha \beta
\end{array}\right\} \frac{d x^{\alpha}}{d s} \frac{d x^{\beta}}{d s}=\frac{e}{m c^{3}}\left(\phi^{\alpha \mu}+F_{-}^{\alpha \mu}\right) u_{\alpha}
$$

here $\left(F_{-}^{\mu \nu}\right)$ is a tensor defined by

$$
F_{-}^{\mu \nu}=\frac{1}{2}\left(F_{\text {ret }}^{\mu \nu}-F_{\text {adv }}^{\mu \nu}\right),
$$

$\left(F_{\text {ret }}^{\mu \nu}\right)$ and $\left(F_{\text {adv }}^{\mu \nu}\right)$ being the retarded and advanced electromagnetic field tensors having the test charge as the source. It is found that $[14, p .221]$

$$
F_{-}^{\mu \nu}={ }_{1} F^{\mu \nu}+{ }_{2} F^{\mu \nu}
$$

where

$$
{ }_{1} F^{\mu \nu}=\frac{2 e}{3 c^{4}}\left(u^{\mu} \dot{a}^{\nu}-u^{\nu} \dot{a}^{\mu}\right)
$$

and

$$
{ }_{2} F^{\mu \nu}=\frac{e}{2} \int_{-\infty}^{\infty} \varepsilon\left(\tau-\tau^{\prime}\right) f_{B^{\prime \nu}} u^{B^{\prime}}\left(\tau^{\prime}\right) d \tau^{\prime}
$$

in which $\varepsilon(\tau)=\tau /|\tau|$. The tensor $\left({ }_{1} F^{\mu \nu}\right)$ is the same as $\left(\dot{s}^{\mu \nu}\right)$, where $\left(s^{\mu \nu}\right)$ is defined by (4). The tensor $\left({ }_{2} F^{\mu \nu}\right)$ involves the bi-tensor introduced by Dewitt and Brehme [4]; $\left({ }_{2} F^{\mu \nu}\right)$ is skew-symmetric.

The equation of motion (59) is of the same form as that found by Dewitt and Brehme [4], but the integral terms differ; there is no term in (j:0) like that found by Hobbs [11]. Equation (59) can be written as the geodesic equation of the affine connection $\Gamma$ with components

$$
\Gamma_{\alpha \beta}^{\mu}=\left\{\begin{array}{c}
\mu \\
\alpha \beta
\end{array}\right\}-\frac{e}{m c^{3}}\left(\phi_{\alpha}^{\mu}+{ }_{1} F_{\alpha}^{\mu}+{ }_{2} F_{\alpha}^{\mu}\right) u_{\beta} \text {. }
$$


Because of the skew-symmetry of $\left(\phi_{\mu \nu}\right),\left({ }_{1} F_{\mu \nu}\right)$ and $\left({ }_{2} F_{\mu \nu}\right)$, the covariant derivative of $\left(g_{\mu \nu}\right)$ with respect to $\Gamma$ vanishes. The torsion vector of $\Gamma$ has components

$$
\Gamma_{\alpha}=\frac{-e}{2 m c^{3}}\left(\phi_{\alpha}^{\sigma}+{ }_{1} F_{\alpha}^{\sigma}+{ }_{2} F_{\alpha}^{\sigma}\right) u_{\sigma}
$$

and is thus proportional to the total 4-force on the particle.

\section{Concluding remarks}

In this paper, the equation of motion of a point test charge in a general Riemannian space-time, with the effect of electromagnetic radiation reaction included, has been expressed as the geodesic equation of an affine connection. The connection is the sum of the usual Christoffel connection and a tensor consisting of several parts. When radiation reaction is neglected, the part of the tensor which remains depends on the external electromagnetic field, the charge-to-mass ratio of the particle and the particle's 4-velocity: the affinity is not an "external" property of space, independent of the particle. The parts of the affinity which arise from the radiation reaction depend on the particle's charge and mass, its 4-velocity and rate of change of 4-acceleration with respect to proper time, the Ricci tensor and a bi-tensor introduced by DeWitt and Brehme [4].

A particular choice of affine connection has been made, so that the covariant derivative of the metric tensor, taken with respect to the resulting affinity, vanishes. A possible alternative condition on the affinity is one which relates to a non-symmetric fundamental tensor, and was used in the unified field theory developed by Einstein, Schrödinger and others $[16 ; 9$, Appendix II; 10]. This condition has previously been applied in the case in which radiation reaction is neglected [3]; its application when radiation reaction is included is a topic for future investigation. 


\section{References}

[1] A.0. Barut, Electrodynomics and classical theory of fields and particles (The MacMillan Company, New York; Collier-MacMillan, London; 1964).

[2] R. Burman, "Particle paths of general relativity as geodesics of an affine connection", Bul2. Austral. Math. Soc. 3 (1970), 325-335.

[3] R.R. Burman, "Paths of charges in general relativity as geodesics of Einstein's non-Riemannian geometry", Bulz. Austral. Math. Soc. 4 (1971), 69-84.

[4] Bryce S. Dewitt and Robert W. Brehme, "Radiation damping in a gravitational field", Ann. Physics 9 (1960), 220-259.

[5] Cécile Morette DeWitt and Bryce S. DeWitt, "Falling charges", Physics 1 (1964), 3-20.

[6] P.A.M. Dirac, "Classical theory of radiating electrons", Proc. Roy. Soc. London Ser. A 167 (1938), 148-169.

[7] P. Droz-Vincent, "Electromagnetism and geodesics", Nuovo Cimento B $51(1967), 555-556$.

[8] A.S. Eddington, The mathematical theory of relativity, 2nd ed. (Cambridge University Press, Cambridge, 1924).

[9] Albert Einstein, The meaning of relativity, 6th ed. (Methuen, London, $1956)$.

[10] Václav Hlavatý, Geometry of E'instein's unified field theory (Noordhoff, Groningen, N.D. [1957]).

[11] J.M. Hobbs, "A vierbein formalism of radiation damping", Ann. Physics 47 (1968), 141-165.

[12] J.M. Hobbs, "Radiation damping in conformally flat universes", Ann. Physics 47 (1968), 166-172.

[13] F. Rohrlich, "Solution of the classical electromagnetic self-energy problem", Phys. Rev. Letters 12 (1964), 375-377.

[14] F. Rohrlich, Classical charged particles. Foundations of their theory (Addison-Wesley, Reading, Massachusetts, 1965). 
[15] F. Rohrlich and J. Winicour, "The equations of motion of a system of charged particles in a gravitational field", Perspectives in geometry and relativity (Essays in honor of Václav Hlavatý, edited by Banesh Hoffmann), 318-324. (Indiana University Press, Bloomington, London, 1966).

[16] Erwin Schrödinger, Space-time structure (Cambridge University Press, Cambridge, 1950).

[17] D.K. Sen, "A simple derivation of the geodesic equations of motion from the matter tensor in general relativity using the

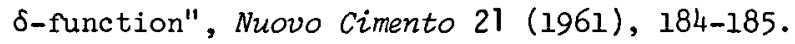

[18] D.K. Sen, Fields and/or particles (The Ryerson Press, Toronto; Academic Press, London, New York, 1968).

[19] J.L. Synge, Relativity: The general theory (North-Holland, Amsterdam; Interscience, New York; 1960).

University of Western Australia, Ned.lands, Western Australia. 